\title{
Gastric Cancer Perforation: Experience from a Tertiary Care Hospital
}

\author{
Bishnu Prasad Kandel,' Yogendra Singh,' Keshav Prasad Singh,' Mahesh Khakurel ${ }^{2}$ \\ 'Department of Surgery, Tribhuvan University Teaching Hospital, ${ }^{2}$ Department of Surgery, KIST Medical College, \\ Kathmandu, Nepal.
}

\section{ABSTRACT}

Introduction: Gastric cancer perforation can occurs in advanced stage of the disease and is often associated with a high morbidity and mortality. Peritonitis due to perforation needs emergency laparotomy and different surgical procedures can be performed for definitive treatment. Surgical procedures largely depend on the stage of the disease and general condition of the patient. This study was carried out to evaluate the outcome and role of different surgical procedures in gastric cancer perforation.

Methods: Medical record of patients with gastric perforation, who were treated during ten years period, was reviewed retrospectively. Data regarding clinical presentation, surgical procedures, staging and survival of patients were obtained.

Results: Features suggestive of diffuse peritonitis were evident in all cases. The majority of the patients underwent emergency surgery except one who died during resuscitation. The majority of patients were in stage III and stage IV. Surgical procedure includes simple closure and omental patch in five patients, simple closure and gastrojejunostomy in nine patients, gastrectomy in six patients and Devine's antral exclusion in one patient. Surgical site infection was the most common (45.5\%) postoperative complication. Four patients died within one month of the surgery. Three patients who underwent gastrectomy survived for one year and one patient survived for five years.

Conclusions: Although gastric cancer perforation usually occurs in advanced stage of the disease, curative resection should be considered as far as possible.

Keywords: gastric cancer; gastrectomy; laparotomy; perforation; peritonitis.

\section{INTRODUCTION}

Perforation peritonitis is a rare complication of gastric cancer. It represents $1 \%$ of patients presenting as diffuse peritonitis. ${ }^{1,2}$ It usually occurs in advanced disease and preoperative diagnosis of gastric cancer perforation is rarely possible. In presence of peritonitis, management of perforated gastric cancer is a challenging surgical emergency. Besides surgical treatment of peritonitis, it needs intraoperative diagnosis, staging and proper oncological surgery. Unresectable diseases can be treated by simple closure with gastrojejunostomy

Correspondence: Dr. Bishnu Prasad Kandel, Department of Surgery, Tribhuvan University Teaching Hospital, Kathmandu, Nepal. Email: drkandel@hotmail.com, Phone: 9841179253. 
if needed; whereas resectable tumors can undergo gastrectomy. The definitive treatment of resectable tumors can be done in one or two stage operation. ${ }^{3}$ But the treatment of choice in perforated gastric cancer still remains uncertain. In this study we reviewed the outcome of different surgical procedure performed in patients with gastric cancer perforation.

\section{METHODS}

This is a retrospective study where we reviewed all the medical records of patients who underwent treatment for gastric carcinoma in Tribhuvan University Teaching Hospital over a period of 10 years, from 1995 to 2005 AD. Data regarding patients' age, sex, preoperative diagnosis, location of perforation, lymph node involvement, surgical procedures done and stage of the tumor were obtained from the medical records and analyzed. Information regarding survival of the patients was obtained from phone calls and follow up records (up to five years).

\section{RESULTS}

Total of 488 gastric cancer patients were subjected to surgical management during that period. Twenty-two of these patients had perforated gastric cancer. All patients were managed as a surgical emergency and laparotomy done with midline incision. Intraoperative diagnosis, surgical staging and decision of resection or palliative procedure were done by consultant surgeon. All patients who underwent gastrectomy, were given adjuvant chemotherapy consisting of six cycles of ECF(Epirubicin, cisplatin and 5- fluorouracil) regimen.

Of 488 patients of carcinoma stomach, 22 (4.5\%) patients had perforation of the cancer. The median age was 65 (Range 40-74) years. Male: female ratio was 1.4:1 and the mean duration of symptoms of peritonitis was 19.5 hours. Twenty of these patients presented in the emergency with features of peritonitis. One patient presented with gastric outlet obstruction and developed perforation while he was being resuscitated in the hospital. Another patient developed perforation peritonitis after few hours of gastroscopic examination. Preoperative diagnosis of gastric cancer perforation was made in these two cases whereas in other patients' diagnosis of hollow viscous perforation most likely duodenal ulcer perforation was made. Chest $X-$ Ray revealed features of free gas under the diaphragm in $17(77.3 \%)$ cases. Twenty one patients underwent emergency laparotomy and one patient with poor general condition expired during resuscitation. Most of the patient $(77.3 \%)$ had ulcerative growth with perforation in the antrum (Figure 1).

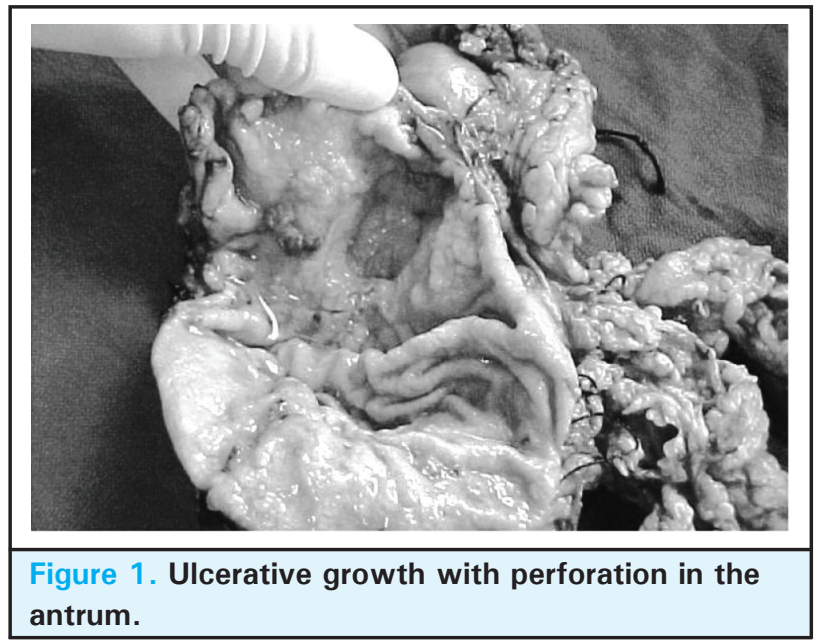

Fifteen $(68.2 \%$ ) patients had advanced disease (stage III or IV) with peritoneal (22.7\%) or liver (50\%) metastasis (Table 1). Five of these patients had tumor on the antrum and there were no features of gastric outlet obstruction and they were treated with simple closure of the perforation. Nine patients had antral tumor and gastrojejunostomy was also done with anticipation of gastric outlet obstruction. Devine's antral exclusion (Modified) was done in one patient with large perforation. Six patients with resectable tumor without peritoneal or liver metastasis were treated with gastrectomy. Five of the gastrectomies were done in one- stage and one in two stage operations. Two-stage gastrectomy was attempted in one other patient but due to dense adhesion resection was not possible. Among these six patients one patient had stage II disease and other five had stage III disease.

\begin{tabular}{|ll|}
\hline \multicolumn{2}{|l|}{ Table 1. Clinicopathological feature of 22 patients } \\
with perforated gastric cancer. \\
\hline Variable & $\mathrm{n}(\%)$ \\
Sex & \\
Male & $13(59.1 \%)$ \\
Female & $9(40.9 \%)$ \\
Preoperative diagnosis & \\
Duodenal Ulcer perforation & $20(90.9 \%)$ \\
Perforated gastric cancer & $2(9.1 \%)$ \\
Tumor location & \\
Antrum & $17(77.3 \%)$ \\
Lesser curvature & $5(22.7 \%)$ \\
Size of perforation & $0.5-3 \mathrm{~cm}$ \\
Histological type & \\
Adenocarcinoma & $22(100 \%)$
\end{tabular}




\begin{tabular}{|ll|} 
Metastasis & \\
Perigastric Lymph nodes & $21(95.5 \%)$ \\
Peritoneal seedling & $5(22.7 \%)$ \\
Liver metastasis & $11(50 \%)$ \\
Stage & \\
I & 0 \\
II & $1(4.5 \%)$ \\
III & $10(45.5 \%)$ \\
IV & $11(50 \%)$ \\
\hline
\end{tabular}

Most common postoperative complication was surgical site infection $(45.5 \%)$ followed by wound dehiscence and anastomotic leakage (Table 2). Four patients with metastasis died within 30 days of operation. Of the six patients who underwent gastrectomy, three survived for one year, two for 2 years and one for 5 years. The mean survival of patients with gastrectomy was 31 months.

\section{Table 2. Surgical Procedures and outcome.}

\begin{tabular}{ll|}
\hline Variables & $\mathbf{n}(\%)$ \\
Surgery & \\
Simple closure only & $5(22.7 \%)$ \\
Closure \& gastrojejunostomy & $9(40.9 \%)$ \\
Modified Devine's antral exclusion & $1(4.5 \%)$ \\
Gastrectomy & $6(27.3 \%)$ \\
Surgery not done & $1(4.5 \%)$ \\
Gastrectomy & \\
One stage & $5(22.7 \%)$ \\
Two stage & $1(4.5 \%)$ \\
Post operative complications & 10 \\
Surgical site infection & $(45.5 \%)$ \\
Wound dehiscence & $1(4.5 \%)$ \\
Anastomotic leake & $1(4.5 \%)$ \\
Perioperative mortality (within 1 & \\
month) & $4(18.2 \%)$ \\
Survival of gastrectomy cases (total & $6(100 \%)$ \\
six) & $3(50 \%)$ \\
One year & $1(16.7 \%)$ \\
Two year & \\
Five year & \\
\hline
\end{tabular}

\section{DISCUSSION}

Perforation is a fatal complication of gastric cancer. Perforation of gastric cancer generally occurs in elderly patients and in advanced stage of the disease when the growth invades all the layers of the stomach wall. ${ }^{4}$ Gastric cancer perforation occurs in $0.75 \%$ to $6 \%$ of gastric cancer patients. ${ }^{1,3}$ The incidence of gastric cancer perforation was higher in series published before 1980 where as in recent literatures the incidence is on lower side. It may be due to technological advances in early detection and management of the disease. But, in the era of endoscopic interventions to cure the early gastric cancer, some patients still present with perforation peritonitis. In our series the incidence was $4.5 \%$, which is higher in comparison to many other series. This may be due to poor access to health care facility and advanced stage at presentation in our part of the world. To the best of our knowledge this is the largest series of gastric cancer perforation from our country.

It is not a common cause of peritonitis and preoperative diagnosis is rarely possible. When a gastric perforation is encountered during laparotomy, malignant perforation should be suspected as $10-16 \%$ of gastric perforation is caused by gastric cancer. ${ }^{3,5,6}$ In presence of diffuse peritonitis intraoperative diagnosis of the cancer is often difficult particularly when frozen section evaluation is not available. Peritoneal seedlings and clinical staging may be misinterpreted due to inflammation. In our series preoperative diagnosis was possible in two patients $(9 \%)$ and frozen section evaluation for emergency operation was not available. Intraoperative diagnosis of malignant lesion was made in 20 (95\%) of the 21 patients. Intraoperative clinical diagnosis has good specificity $(93.8 \%)$ but low sensitivity $(50-60 \%))^{4,7}$ From data of 67 patients with gastric cancer perforation, Ergul et al suggested perforation is malignant if age more than 60 years, an ulcer diameter (with edema) more than $6 \mathrm{~cm}$, a perforation diameter more than $0.5 \mathrm{~cm}$, a symptom duration of more than 20 hour, and a white blood cell count less than $15.103 / \mu \mathrm{L} .{ }^{8}$ This system has a specificity of $98.7 \%$, a sensitivity of $53.7 \%$, a negative predicted value of $93.4 \%$, and positive predicted value of $85.7 \% .^{8}$ In our series, in one patient the lesion was considered benign at first operation and gastrectomy was done after histological diagnosis of malignancy.

Perforation usually occurs in advanced cancer, but it can also occur in early disease. ${ }^{9}$ Adachi et al reported 155 cases of gastric cancer perforation, of which $19 \%$ had stage I and $12 \%$ had stage II cancer. ${ }^{10}$ The perforated gastric cancer is usually found in the greater curvature and anterior wall but those with bleeding are found in lesser curvature. ${ }^{11}$ In our study majority of the cancer were in greater curvature and anterior wall of antral region.

These patients are generally ill with peritonitis in addition to advanced stage of the disease. Peritonitis needs emergency surgery to clear the peritoneal cavity and stop the source of peritonitis whereas gastric cancer needs definitive oncological surgery. Performing extensive resection in unprepared patient in emergency setup may increase the morbidity. Only palliative procedures 
can be performed in advanced disease but patients with resectable tumors benefit more after curative surgery. ${ }^{4}$ At the time of initial surgery radical gastrectomy with lymphadenectomy may not be possible, either because a diagnosis of gastric cancer is not confirmed or because the patient's condition does not allow extended surgery. ${ }^{6}$ Unstable patients with resectable tumor may be good candidate for two stage operation which consist of procedures to deal with perforation and peritonitis at first operation and gastrectomy and lymphadenectomy in later date after patients recovery and histological conformation. ${ }^{6}$ Tumor staging can be completed when the patient has recovered and a radical operation can be performed without compromising long-term prognosis. ${ }^{6,12}$ But second operation after peritonitis might be difficult. In one of our patients the definitive procedure was not possible due to dense adhesions. Patients who underwent curative resection has longer survival rate. ${ }^{13-15}$ In our study most of the patients had advanced disease and curative resection was possible in only six patients. Curative resections in most of our patients were done in one stage operation.

Perforated gastric cancer usually have poor prognosis with mortality within 30 days ranges from $8 \%$ to $52 \% .{ }^{11,16}$ In our study four patients $(18 \%)$ died within 30 days. Perforation may lead to further peritoneal dissemination of the malignant cells. ${ }^{13}$ But peritoneal

\section{REFERENCES}

1. Kasakura Y, Ajani JA, Fijii M, Mochizuki F, Takayama T. Management of Perforated Gastric Carcinoma: A Report of 16 Cases and Review of World Literature. Am Surg. 2002;68:434-40.

2. Kotan C, Sumer A, Baser M, Kizıltan R, Carparlar MA. An Analysis of 13 Patients with Perforated Gastric Carcinoma: A Surgeon's Nightmare? World J Emerg Surg. 2008;3:17-22.

3. Rovillo F, Rossi S, Marrelli D, De Manzoni G, Pedrazzani C, Morgagni P et al. Perforated Gastric Carcinoma : Report of 10 Cases and Review of Literature. World J Surg Oncol. 2006;4:19-24.

4. Jwo SC, Chien RN, Chao TC, Chen YH, Lin CY. Clinicopathological Features, Surgical Management, and Disease Outcome of Perforated Gastric Cancer. J Surg Oncol. 2005;91:219-25.

5. Miura T, Ishii $\mathrm{T}$, Shimoyama $\mathrm{T}$, Hirano $\mathrm{T}$, Tomita M. Surgical Treatment of Perforated Gastric Cancer. Dig Surg.1985;2:200-4.

6. Lehnert T, Buhl K, Dueck M, Hinz U, Herfarth C. Two-staged Radical Gastrectomy for Perforated Gastric Cancer. Eur J Surg Oncol. 2000;26:780-4. metastasis due to cancer perforation may not cause significant problem as it does not influence the survival of the patients. ${ }^{10}$ Perforation of gastric cancer does not preclude long-term survival per se in a substantial number of patients. ${ }^{5,6}$ In patients who underwent curative resection survival rate is influenced by the stage of the disease and it is similar to those without perforation and underwent elective surgery. ${ }^{10,17}$

Lee et al found that potentially curative resection; TNM stage, and the absence of postoperative complications are the important factors associated with improved survival. ${ }^{11}$ Diffuse peritonitis, difficulties and errors of diagnosis and the period between perforation and operation are also important factors determining the prognosis. ${ }^{16,18}$ Perioperative mortality of patients who are operated for gastric cancer is higher than that of patients who underwent emergency surgery for benign ulcer. ${ }^{19}$ Major surgical complication includes surgical site infection, anastomotic leakage and intestinal fistula. ${ }^{20}$ In addition, in patients with perforation, peritoneal recurrence occurs more frequently. ${ }^{7,20}$

\section{CONCLUSIONS}

Perforation peritonitis is a rare complication of gastric cancer associated with high postoperative morbidity and mortality. Perforation usually occurs in advanced stage of the disease but long-term survival can be expected in patients who underwent curative resection.

7. Tsujimoto H, Hiraki S, Sakamoto N, Yaguchi Y, Horio T, Kumano I et al. Outcome After Emergency Surgery in Patients With a Free Perforation Caused by Gastric Cancer. Expernt Therapt Med. 2010;1:199-203.

8. Ergul E, Gozetlik EO. Emergency Spontaneous Gastric Perforations: Ulcer Versus Cancer. Langenbeck's Archiv Surg. 2009;394:643-4.

9. Mukai M, Kondou Y, Ogoshi K, Noto T, makuuchi H, Tajima T. A Case of Perforated Early Gastric Cancer and a Review of 45 Cases Collected from Japanese Literature. J Jpn Soc Clin Surg.1992;53:1869-73

10. Adachi Y, Mori M, Maehara Y, Mastsumata T, Okudaira Y, Sugimachi K. Surgical Results of Perforated Gastric Cancinoma: An Analysis of 155 Japanese Patients. Am J Gastroenterol.1997;92:516-8.

11. Lee HJ, Park DJ, Yang HK, Lee KU, Choe KJ. Outcome after Emergency Surgery in Gastric Cancer Patients with free Perforation or Severe Bleeding. Dig Surg. 2006;23:217-23.

12. Wakato U, Hiroshi K, Hideki M, Hisashi N, Nobuhiko A Yoichi $\mathrm{N}$ et al. A Study of Perforation of Gastric Cancer. J Nihon Univ Med Asso. 2001;60:24-8. 
13. Kasakura Y, Ajani JA, Mochizuki F, Morishita Y,Fijii M, Takayama T. Outcomes after Emergency Surgery for Gastric Perforation or Severe Bleeding in Patients With Gastric Cancer. J Surg Oncol. 2002;80:181-5.

14. Stechenberg L, Bunch RH, Anderson MC. The Surgical Therapy for Perforated Gastric Cancer. Am Surg.1981;47:208-10.

15. Gertsch P, Yip SKH, Chow LWC, Lauder IJ. Free Perforation of Gastric Carcinoma: Results of Surgical Treatment. Arch Surg. 1995;130:177-181

16. Anishin NS. Perforation of Gastric Cancer. Vestn Khir Im Grek. 1976;116:30-4.
17. Adachi Y, Aramaki M, Shirishi N, Shimoda K, Yashoda K, Kitano S. Long-term Survival after Perforation of Advanced Gastric Cancer: Case Report and Review of Literature. Gastric Cancer. 1998;1:80-3.

18. Kamer E, Unalp HR, Atahan K, Peşkersoy M, Tarcan E, Onal MA: Outcomes of Surgical Therapy and Prognostic Factors in Spontaneous Gastric Tumor Perforation. Ulus Travma Acil Cerrahi Derg. _2009;15:571-8

19. So JBY, Yam A, Cheah WK, Kum CK, Goh PM. Risk Factors Related to Operative Mortality and Morbidity in Patients Undergoing Emergency Gastrectomy. Br J Surg. 2000;87:1702-7.

20. Yildirim Y, Erkan N, Avci E, Elveren B. Perforated Gastric Cancer Complicating Early Postpartum Period of Pregnancy. Acta Chir Belg. 2009;109:534-7. 